\title{
Search for Long-Lived Particles Decaying into Electron or Photon Pairs with the D0 Detector
}

V. M. Abazov,${ }^{36}$ B. Abbott,${ }^{75}$ M. Abolins, ${ }^{65}$ B. S. Acharya, ${ }^{29}$ M. Adams,${ }^{51}$ T. Adams, ${ }^{49}$ E. Aguilo, ${ }^{6}$ M. Ahsan, ${ }^{59}$ G. D. Alexeev, ${ }^{36}$ G. Alkhazov, ${ }^{40}$ A. Alton, ${ }^{64, *}$ G. Alverson, ${ }^{63}$ G. A. Alves, ${ }^{2}$ M. Anastasoaie, ${ }^{35}$ L. S. Ancu, ${ }^{35}$ T. Andeen, ${ }^{53}$ B. Andrieu, ${ }^{17}$ M. S. Anzelc, ${ }^{53}$ M. Aoki,${ }^{50}$ Y. Arnoud,${ }^{14}$ M. Arov, ${ }^{60}$ M. Arthaud,${ }^{18}$ A. Askew, ${ }^{49}$ B. Asman,${ }^{41}$

A. C. S. Assis Jesus, ${ }^{3}$ O. Atramentov, ${ }^{49}$ C. Avila, ${ }^{8}$ F. Badaud,${ }^{13}$ L. Bagby,${ }^{50}$ B. Baldin,${ }^{50}$ D. V. Bandurin, ${ }^{59}$ P. Banerjee, ${ }^{29}$ S. Banerjee, ${ }^{29}$ E. Barberis ${ }^{63}$ A.-F. Barfuss,${ }^{15}$ P. Bargassa,${ }^{80}$ P. Baringer, ${ }^{58}$ J. Barreto, ${ }^{2}$ J. F. Bartlett ${ }^{50}$ U. Bassler, ${ }^{18}$

D. Bauer, ${ }^{43}$ S. Beale ${ }^{6}$ A. Bean, ${ }^{58}$ M. Begalli, ${ }^{3}$ M. Begel,${ }^{73}$ C. Belanger-Champagne, ${ }^{41}$ L. Bellantoni, ${ }^{50}$ A. Bellavance,${ }^{50}$ J. A. Benitez, ${ }^{65}$ S. B. Berii, ${ }^{27}$ G. Bernardi, ${ }^{17}$ R. Bernhard, ${ }^{23}$ I. Bertram,${ }^{42}$ M. Besançon, ${ }^{18}$ R. Beuselinck,${ }^{43}$ V. A. Bezzubov, ${ }^{39}$ P.C. Bhat ${ }^{50}$ V. Bhatnagar, ${ }^{27}$ C. Biscarat,${ }^{20}$ G. Blazey, ${ }^{52}$ F. Blekman ${ }^{43}$ S. Blessing,${ }^{49} \mathrm{~K}$. Bloom,${ }^{67}$ A. Boehnlein,${ }^{50}$ D. Boline, ${ }^{62}$ T. A. Bolton, ${ }^{59}$ E. E. Boos, ${ }^{38}$ G. Borissov, ${ }^{42}$ T. Bose, ${ }^{77}$ A. Brandt,${ }^{78}$ R. Brock, ${ }^{65}$ G. Brooijmans, ${ }^{70}$ A. Bross, ${ }^{50}$ D. Brown,${ }^{81}$ X. B. Bu, ${ }^{7}$ N. J. Buchanan, ${ }^{49}$ D. Buchholz,${ }^{53}$ M. Buehler, ${ }^{81}$ V. Buescher ${ }^{22}$ V. Bunichev, ${ }^{38}$ S. Burdin, ${ }^{42,+}$ T. H. Burnett ${ }^{82}$ C. P. Buszello, ${ }^{43}$ J. M. Butler, ${ }^{62}$ P. Calfayan, ${ }^{25}$ S. Calvet, ${ }^{16}$ J. Cammin,${ }^{71}$ E. Carrera, ${ }^{49}$ W. Carvalho, ${ }^{3}$ B. C. K. Casey, ${ }^{50}$ H. Castilla-Valdez, ${ }^{33}$ S. Chakrabarti, ${ }^{18}$ D. Chakraborty, ${ }^{52}$ K. M. Chan,${ }^{55}$ A. Chandra, ${ }^{48}$ E. Cheu, ${ }^{45}$ F. Chevallier, ${ }^{14}$ D. K. Cho, ${ }^{62}$ S. Choi, ${ }^{32}$ B. Choudhary, ${ }^{28}$ L. Christofek,${ }^{77}$ T. Christoudias, ${ }^{43}$ S. Cihangir, ${ }^{50}$ D. Claes, ${ }^{67}$ J. Clutter, ${ }^{58}$ M. Cooke, ${ }^{50}$ W. E. Cooper, ${ }^{50}$ M. Corcoran, ${ }^{80}$ F. Couderc, ${ }^{18}$ M.-C. Cousinou, ${ }^{15}$ S. Crépé-Renaudin, ${ }^{14}$ V. Cuplov, ${ }^{59}$ D. Cutts, ${ }^{77}$ M. Ćwiok, ${ }^{30}$ H. da Motta, ${ }^{2}$ A. Das, ${ }^{45}$ G. Davies, ${ }^{43}$ K. De,${ }^{78}$ S. J. de Jong,${ }^{35}$ E. De La Cruz-Burelo, ${ }^{33}$ C. De Oliveira Martins, ${ }^{3}$ K. DeVaughan, ${ }^{67}$ J. D. Degenhardt, ${ }^{64}$ F. Déliot, ${ }^{18}$ M. Demarteau, ${ }^{50}$ R. Demina, ${ }^{71}$ D. Denisov, ${ }^{50}$ S. P. Denisov, ${ }^{39}$ S. Desai,${ }^{50}$ H. T. Diehl,${ }^{50}$ M. Diesburg, ${ }^{50}$ A. Dominguez,${ }^{67}$ H. Dong, ${ }^{72}$ T. Dorland, ${ }^{82}$ A. Dubey, ${ }^{28}$ L. V. Dudko, ${ }^{38}$ L. Duflot, ${ }^{16}$ S. R. Dugad,${ }^{29}$ D. Duggan, ${ }^{49}$ A. Duperrin, ${ }^{15}$ J. Dyer, ${ }^{65}$ A. Dyshkant, ${ }^{52}$ M. Eads,${ }^{67}$ D. Edmunds, ${ }^{65}$ J. Ellison, ${ }^{48}$ V. D. Elvira, ${ }^{50}$ Y. Enari, ${ }^{77}$ S. Eno, ${ }^{61}$ P. Ermolov, ${ }^{38, * *}$ H. Evans, ${ }^{54}$ A. Evdokimov ${ }^{73}$ V. N. Evdokimov, ${ }^{39}$ A. V. Ferapontov, ${ }^{59}$ T. Ferbel,,${ }^{71}$ F. Fiedler,${ }^{24}$ F. Filthaut,${ }^{35}$ W. Fisher,${ }^{50}$ H. E. Fisk, ${ }^{50}$ M. Fortner,${ }^{52}$ H. Fox,${ }^{42}$ S. Fu, ${ }^{50}$ S. Fuess, ${ }^{50}$ T. Gadfort, ${ }^{70}$ C. F. Galea,${ }^{35}$ C. Garcia, ${ }^{71}$ A. Garcia-Bellido, ${ }^{71}$ V. Gavrilov, ${ }^{37}$ P. Gay, ${ }^{13}$ W. Geist ${ }^{19}$ W. Geng, ${ }^{15,65}$ C. E. Gerber, ${ }^{51}$ Y. Gershtein, ${ }^{49}$ D. Gillberg, ${ }^{6}$ G. Ginther ${ }^{71}$ N. Gollub,${ }^{41}$ B. Gómez, ${ }^{8}$ A. Goussiou, ${ }^{82}$ P. D. Grannis, ${ }^{72}$ H. Greenlee, ${ }^{50}$ Z. D. Greenwood,${ }^{60}$ E. M. Gregores,${ }^{4}$ G. Grenier ${ }^{20} \mathrm{Ph}$. Gris, ${ }^{13}$ J.-F. Grivaz, ${ }^{16}$ A. Grohsjean, ${ }^{25}$ S. Grünendahl,${ }^{50}$ M. W. Grünewald ${ }^{30}$ F. Guo, ${ }^{72}$ J. Guo, ${ }^{72}$ G. Gutierrez,${ }^{50}$ P. Gutierrez,${ }^{75}$ A. Haas, ${ }^{70}$ N. J. Hadley, ${ }^{61}$ P. Haefner, ${ }^{25}$ S. Hagopian, ${ }^{49}$ J. Haley, ${ }^{68}$ I. Hall, ${ }^{65}$ R. E. Hall, ${ }^{47}$ L. Han, ${ }^{7}$ K. Harder, ${ }^{44}$ A. Harel,${ }^{71}$ J. M. Hauptman, ${ }^{57}$ J. Hays, ${ }^{43}$ T. Hebbeker, ${ }^{21}$ D. Hedin, ${ }^{52}$ J. G. Hegeman,${ }^{34}$ A. P. Heinson, ${ }^{48}$ U. Heintz,${ }^{62}$ C. Hensel, ${ }^{22,8}$ K. Herner, ${ }^{72}$ G. Hesketh, ${ }^{63}$ M. D. Hildreth, ${ }^{55}$ R. Hirosky, ${ }^{81}$ J. D. Hobbs, ${ }^{72}$ B. Hoeneisen, ${ }^{12}$ H. Hoeth, ${ }^{26}$ M. Hohlfeld, ${ }^{22}$ S. Hossain, ${ }^{75}$ P. Houben, ${ }^{34}$ Y. Hu, ${ }^{72}$ Z. Hubacek, ${ }^{10}$ V. Hynek, ${ }^{9}$ I. Iashvili, ${ }^{69}$ R. Illingworth,${ }^{50}$ A. S. Ito, ${ }^{50}$ S. Jabeen, ${ }^{62}$ M. Jaffré, ${ }^{16}$ S. Jain, ${ }^{75}$ K. Jakobs, ${ }^{23}$ C. Jarvis, ${ }^{61}$ R. Jesik, ${ }^{43}$ K. Johns, ${ }^{45}$ C. Johnson, ${ }^{70}$ M. Johnson, ${ }^{50}$ D. Johnston, ${ }^{67}$ A. Jonckheere, ${ }^{50}$ P. Jonsson, ${ }^{43}$ A. Juste, ${ }^{50}$ E. Kajfasz, ${ }^{15}$ J. M. Kalk ${ }^{60}$ D. Karmanov, ${ }^{38}$ P. A. Kasper, ${ }^{50}$ I. Katsanos, ${ }^{70}$ D. Kau, ${ }^{49}$ V. Kaushik, ${ }^{78}$ R. Kehoe, ${ }^{79}$ S. Kermiche, ${ }^{15}$ N. Khalatyan, ${ }^{50}$ A. Khanov, ${ }^{76}$ A. Kharchilava, ${ }^{69}$ Y. M. Kharzheev, ${ }^{36}$ D. Khatidze ${ }^{70}$ T. J. Kim ${ }^{31}$ M. H. Kirby, ${ }^{53}$ M. Kirsch, ${ }^{21}$ B. Klima, ${ }^{50}$ J. M. Kohli, ${ }^{27}$ J.-P. Konrath, ${ }^{23}$ A. V. Kozelov ${ }^{39}$

J. Kraus, ${ }^{65}$ T. Kuhl,${ }^{24}$ A. Kumar, ${ }^{69}$ A. Kupco, ${ }^{11}$ T. Kurča,${ }^{20}$ V. A. Kuzmin, ${ }^{38}$ J. Kvita, ${ }^{9}$ F. Lacroix, ${ }^{13}$ D. Lam, ${ }^{55}$

S. Lammers, ${ }^{70}$ G. Landsberg, ${ }^{77}$ P. Lebrun, ${ }^{20}$ W. M. Lee, ${ }^{50}$ A. Leflat, ${ }^{38}$ J. Lellouch, ${ }^{17}$ J. Li, ${ }^{78, * *}$ L. Li,${ }^{48}$ Q. Z. Li, ${ }^{50}$ S. M. Lietti, ${ }^{5}$ J. K. Lim, ${ }^{31}$ J. G. R. Lima,${ }^{52}$ D. Lincoln,${ }^{50}$ J. Linnemann,${ }^{65}$ V. V. Lipaev,${ }^{39}$ R. Lipton, ${ }^{50}$ Y. Liu, ${ }^{7}$ Z. Liu ${ }^{6}$ A. Lobodenko, ${ }^{40}$ M. Lokajicek, ${ }^{11}$ P. Love, ${ }^{42}$ H. J. Lubatti, ${ }^{82}$ R. Luna, ${ }^{3}$ A. L. Lyon, ${ }^{50}$ A. K. A. Maciel, ${ }^{2}$ D. Mackin, ${ }^{80}$ R. J. Madaras, ${ }^{46}$ P. Mättig, ${ }^{26}$ C. Magass, ${ }^{21}$ A. Magerkurth, ${ }^{64}$ P. K. Mal, ${ }^{82}$ H. B. Malbouisson, ${ }^{3}$ S. Malik, ${ }^{67}$ V. L. Malyshev, ${ }^{36}$ Y. Maravin, ${ }^{59}$ B. Martin, ${ }^{14}$ R. McCarthy, ${ }^{72}$ A. Melnitchouk, ${ }^{66}$ L. Mendoza, ${ }^{8}$ P. G. Mercadante, ${ }^{5}$ M. Merkin,${ }^{38}$ K. W. Merritt, ${ }^{50}$ A. Meyer, ${ }^{21}$ J. Meyer, ${ }^{22,8}$ J. Mitrevski, ${ }^{70}$ R. K. Mommsen, ${ }^{44}$ N. K. Mondal,${ }^{29}$ R. W. Moore, ${ }^{6}$ T. Moulik, ${ }^{58}$ G. S. Muanza, ${ }^{20}$ M. Mulhearn, ${ }^{70}$ O. Mundal, ${ }^{22}$ L. Mundim,${ }^{3}$ E. Nagy, ${ }^{15}$ M. Naimuddin, ${ }^{50}$ M. Narain, ${ }^{77}$ N. A. Naumann, ${ }^{35}$ H. A. Neal, ${ }^{64}$ J. P. Negret, ${ }^{8}$ P. Neustroev,${ }^{40}$ H. Nilsen, ${ }^{23}$ H. Nogima, ${ }^{3}$ S. F. Novaes, ${ }^{5}$ T. Nunnemann,,${ }^{25}$ V. O'Dell, ${ }^{50}$

D. C. O'Neil, ${ }^{6}$ G. Obrant, ${ }^{40}$ C. Ochando, ${ }^{16}$ D. Onoprienko, ${ }^{59}$ N. Oshima ${ }^{50}$ N. Osman,${ }^{43}$ J. Osta, ${ }^{55}$ R. Otec, ${ }^{10}$

G. J. Otero y Garzón, ${ }^{50}$ M. Owen, ${ }^{44}$ P. Padley, ${ }^{80}$ M. Pangilinan, ${ }^{77}$ N. Parashar, ${ }^{56}$ S.-J. Park ${ }^{22,8}$ S. K. Park, ${ }^{31}$ J. Parsons, ${ }^{70}$ R. Partridge ${ }^{77}$ N. Parua,${ }^{54}$ A. Patwa,${ }^{73}$ G. Pawloski,${ }^{80}$ B. Penning, ${ }^{23}$ M. Perfilov,${ }^{38}$ K. Peters, ${ }^{44}$ Y. Peters,${ }^{26}$ P. Pétroff, ${ }^{16}$ M. Petteni, ${ }^{43}$ R. Piegaia, ${ }^{1}$ J. Piper, ${ }^{65}$ M.-A. Pleier, ${ }^{22}$ P. L. M. Podesta-Lerma, ${ }^{33,}$ V. M. Podstavkov, ${ }^{50}$ Y. Pogorelov, ${ }^{55}$ M.-E. Pol, ${ }^{2}$ P. Polozov,${ }^{37}$ B. G. Pope,${ }^{65}$ A. V. Popov,${ }^{39}$ C. Potter,${ }^{6}$ W. L. Prado da Silva, ${ }^{3}$ H. B. Prosper, ${ }^{49}$ S. Protopopescu, ${ }^{73}$ J. Qian, ${ }^{64}$ A. Quadt, ${ }^{22,8}$ B. Quinn, ${ }^{66}$ A. Rakitine, ${ }^{42}$ M. S. Rangel, ${ }^{2}$ K. Ranjan, ${ }^{28}$ P. N. Ratoff, ${ }^{42}$ P. Renkel,${ }^{79}$ P. Rich, ${ }^{44}$ J. Rieger, ${ }^{54}$ M. Rijssenbeek, ${ }^{72}$ I. Ripp-Baudot, ${ }^{19}$ F. Rizatdinova, ${ }^{76}$ S. Robinson, ${ }^{43}$ R. F. Rodrigues, ${ }^{3}$ M. Rominsky ${ }^{75}$ 
C. Royon, ${ }^{18}$ P. Rubinov, ${ }^{50}$ R. Ruchti, ${ }^{55}$ G. Safronov,${ }^{37}$ G. Sajot, ${ }^{14}$ A. Sánchez-Hernández, ${ }^{33}$ M. P. Sanders,${ }^{17}$ B. Sanghi, ${ }^{50}$ G. Savage, ${ }^{50}$ L. Sawyer ${ }^{60}$ T. Scanlon, ${ }^{43}$ D. Schaile, ${ }^{25}$ R. D. Schamberger, ${ }^{72}$ Y. Scheglov, ${ }^{40}$ H. Schellman,${ }^{53}$

T. Schliephake, ${ }^{26}$ S. Schlobohm,${ }^{82}$ C. Schwanenberger, ${ }^{44}$ A. Schwartzman, ${ }^{68}$ R. Schwienhorst, ${ }^{65}$ J. Sekaric, ${ }^{49}$ H. Severini, ${ }^{75}$ E. Shabalina, ${ }^{51}$ M. Shamim, ${ }^{59}$ V. Shary, ${ }^{18}$ A. A. Shchukin,${ }^{39}$ R. K. Shivpuri, ${ }^{28}$ V. Siccardi, ${ }^{19}$ V. Simak, ${ }^{10}$ V. Sirotenko, ${ }^{50}$ P. Skubic, ${ }^{75}$ P. Slattery, ${ }^{71}$ D. Smirnov, ${ }^{55}$ G. R. Snow, ${ }^{67}$ J. Snow,${ }^{74}$ S. Snyder,${ }^{73}$ S. Söldner-Rembold, ${ }^{44}$ L. Sonnenschein, ${ }^{17}$ A. Sopczak,${ }^{42}$ M. Sosebee, ${ }^{78}$ K. Soustruznik, ${ }^{9}$ B. Spurlock,${ }^{78}$ J. Stark, ${ }^{14}$ J. Steele, ${ }^{60}$ V. Stolin, ${ }^{37}$ D. A. Stoyanova, ${ }^{39}$ J. Strandberg, ${ }^{64}$ S. Strandberg, ${ }^{41}$ M. A. Strang, ${ }^{69}$ E. Strauss, ${ }^{72}$ M. Strauss,${ }^{75}$ R. Ströhmer, ${ }^{25}$ D. Strom, ${ }^{53}$ L. Stutte, ${ }^{50}$ S. Sumowidagdo, ${ }^{49}$ P. Svoisky, ${ }^{55}$ A. Sznajder, ${ }^{3}$ P. Tamburello, ${ }^{45}$ A. Tanasijczuk, ${ }^{1}$ W. Taylor, ${ }^{6}$ B. Tiller, ${ }^{25}$ F. Tissandier, ${ }^{13}$ M. Titov, ${ }^{18}$ V. V. Tokmenin, ${ }^{36}$ I. Torchiani, ${ }^{23}$ D. Tsybychev, ${ }^{72}$ B. Tuchming, ${ }^{18}$ C. Tully, ${ }^{68}$ P. M. Tuts ${ }^{70}$ R. Unalan, ${ }^{65}$ L. Uvarov, ${ }^{40}$ S. Uvarov,${ }^{40}$ S. Uzunyan, ${ }^{52}$ B. Vachon, ${ }^{6}$ P. J. van den Berg, ${ }^{34}$ R. Van Kooten, ${ }^{54}$

W. M. van Leeuwen, ${ }^{34}$ N. Varelas,${ }^{51}$ E. W. Varnes, ${ }^{45}$ I. A. Vasilyev, ${ }^{39}$ P. Verdier, ${ }^{20}$ L. S. Vertogradov,${ }^{36}$ M. Verzocchi, ${ }^{50}$ D. Vilanova, ${ }^{18}$ F. Villeneuve-Seguier, ${ }^{43}$ P. Vint, ${ }^{43}$ P. Vokac, ${ }^{10}$ M. Voutilainen,${ }^{67, \|}$ R. Wagner,${ }^{68}$ H. D. Wahl, ${ }^{49}$

M. H. L. S. Wang, ${ }^{50}$ J. Warchol, ${ }^{55}$ G. Watts, ${ }^{82}$ M. Wayne, ${ }^{55}$ G. Weber, ${ }^{24}$ M. Weber, ${ }^{50,}{ }^{, / 1}$ L. Welty-Rieger, ${ }^{54}$ A. Wenger, ${ }^{23,++}$ N. Wermes, ${ }^{22}$ M. Wetstein, ${ }^{61}$ A. White, ${ }^{78}$ D. Wicke, ${ }^{26}$ M. Williams, ${ }^{42}$ G. W. Wilson, ${ }^{58}$ S. J. Wimpenny,${ }^{48}$ M. Wobisch,${ }^{60}$ D. R. Wood, ${ }^{63}$ T. R. Wyatt, ${ }^{44}$ Y. Xie, ${ }^{77}$ S. Yacoob,${ }^{53}$ R. Yamada ${ }^{50}$ W. -C. Yang, ${ }^{44}$ T. Yasuda,${ }^{50}$ Y. A. Yatsunenko, ${ }^{36}$ H. Yin, ${ }^{7}$ K. Yip ${ }^{73}$ H. D. Yoo, ${ }^{77}$ S. W. Youn, ${ }^{53}$ J. Yu, ${ }^{78}$ C. Zeitnitz, ${ }^{26}$ S. Zelitch, ${ }^{81}$ T. Zhao, ${ }^{82}$ B. Zhou, ${ }^{64}$ J. Zhu, ${ }^{72}$ M. Zielinski, ${ }^{71}$ D. Zieminska, ${ }^{54}$ A. Zieminski, ${ }^{54 * *}$ L. Zivkovic, ${ }^{70}$ V. Zutshi, ${ }^{52}$ and E. G. Zverev ${ }^{38}$

(D0 Collaboration)

\author{
${ }^{1}$ Universidad de Buenos Aires, Buenos Aires, Argentina \\ ${ }^{2}$ LAFEX, Centro Brasileiro de Pesquisas Físicas, Rio de Janeiro, Brazil \\ ${ }^{3}$ Universidade do Estado do Rio de Janeiro, Rio de Janeiro, Brazil \\ ${ }^{4}$ Universidade Federal do ABC, Santo André, Brazil \\ ${ }^{5}$ Instituto de Física Teórica, Universidade Estadual Paulista, São Paulo, Brazil \\ ${ }^{6}$ University of Alberta, Edmonton, Alberta, Canada, \\ Simon Fraser University, Burnaby, British Columbia, Canada, \\ York University, Toronto, Ontario, Canada, \\ and McGill University, Montreal, Quebec, Canada \\ ${ }^{7}$ University of Science and Technology of China, Hefei, People's Republic of China \\ ${ }^{8}$ Universidad de los Andes, Bogotá, Colombia \\ ${ }^{9}$ Center for Particle Physics, Charles University, Prague, Czech Republic \\ ${ }^{10}$ Czech Technical University, Prague, Czech Republic \\ ${ }^{11}$ Center for Particle Physics, Institute of Physics, Academy of Sciences of the Czech Republic, Prague, Czech Republic \\ ${ }^{12}$ Universidad San Francisco de Quito, Quito, Ecuador \\ ${ }^{13}$ LPC, Université Blaise Pascal, CNRS/IN2P3, Clermont, France \\ ${ }^{14}$ LPSC, Université Joseph Fourier Grenoble 1, CNRS/IN2P3, Institut National Polytechnique de Grenoble, Gremoble, France \\ ${ }^{15}$ CPPM, Aix-Marseille Université, CNRS/IN2P3, Marseille, France \\ ${ }^{16}$ LAL, Université Paris-Sud, IN2P3/CNRS, Orsay, France \\ ${ }^{17}$ LPNHE, IN2P3/CNRS, Universités Paris VI and VII, Paris, France \\ ${ }^{18}$ DAPNIA/Service de Physique des Particules, CEA, Irfu, SPP, Saclay, France \\ ${ }^{19}$ IPHC, Université Louis Pasteur, CNRS/IN2P3, Strasbourg, France \\ ${ }^{20} I P N L$, Université Lyon 1, CNRS/IN2P3, Villeurbanne, France and Université de Lyon, Lyon, France \\ ${ }^{21}$ III. Physikalisches Institut A, RWTH Aachen University, Aachen, Germany \\ ${ }^{22}$ Physikalisches Institut, Universität Bonn, Bonn, Germany \\ ${ }^{23}$ Physikalisches Institut, Universität Freiburg, Freiburg, Germany \\ ${ }^{24}$ Institut für Physik, Universität Mainz, Mainz, Germany \\ ${ }^{25}$ Ludwig-Maximilians-Universität München, München, Germany \\ ${ }^{26}$ Fachbereich Physik, University of Wuppertal, Wuppertal, Germany \\ ${ }^{27}$ Panjab University, Chandigarh, India \\ ${ }^{28}$ Delhi University, Delhi, India \\ ${ }^{29}$ Tata Institute of Fundamental Research, Mumbai, India \\ ${ }^{30}$ University College Dublin, Dublin, Ireland \\ ${ }^{31}$ Korea Detector Laboratory, Korea University, Seoul, Korea \\ ${ }^{32}$ SungKyunKwan University, Suwon, Korea \\ ${ }^{33}$ CINVESTAV, Mexico City, Mexico \\ ${ }^{34}$ FOM-Institute NIKHEF and University of Amsterdam/NIKHEF, Amsterdam, The Netherlands
}


${ }^{35}$ Radboud University Nijmegen/NIKHEF, Nijmegen, The Netherlands

${ }^{36}$ Joint Institute for Nuclear Research, Dubna, Russia

${ }^{37}$ Institute for Theoretical and Experimental Physics, Moscow, Russia

${ }^{38}$ Moscow State University, Moscow, Russia

${ }^{39}$ Institute for High Energy Physics, Protvino, Russia

${ }^{40}$ Petersburg Nuclear Physics Institute, St. Petersburg, Russia

${ }^{41}$ Lund University, Lund, Sweden, Royal Institute of Technology and Stockholm University, Stockholm, Sweden, and Uppsala University, Uppsala, Sweden

${ }^{42}$ Lancaster University, Lancaster, United Kingdom

${ }^{43}$ Imperial College, London, United Kingdom

${ }^{44}$ University of Manchester, Manchester, United Kingdom

${ }^{45}$ University of Arizona, Tucson, Arizona 85721, USA

${ }^{46}$ Lawrence Berkeley National Laboratory and University of California, Berkeley, California 94720, USA

${ }^{47}$ California State University, Fresno, California 93740, USA

${ }^{48}$ University of California, Riverside, California 92521, USA

${ }^{49}$ Florida State University, Tallahassee, Florida 32306, USA

${ }^{50}$ Fermi National Accelerator Laboratory, Batavia, Illinois 60510, USA

${ }^{51}$ University of Illinois at Chicago, Chicago, Illinois 60607, USA

${ }^{52}$ Northern Illinois University, DeKalb, Illinois 60115, USA

${ }^{53}$ Northwestern University, Evanston, Illinois 60208, USA

${ }^{54}$ Indiana University, Bloomington, Indiana 47405, USA

${ }^{55}$ University of Notre Dame, Notre Dame, Indiana 46556, USA

${ }^{56}$ Purdue University Calumet, Hammond, Indiana 46323, USA

${ }^{57}$ Iowa State University, Ames, Iowa 50011, USA

${ }^{58}$ University of Kansas, Lawrence, Kansas 66045, USA

${ }^{59}$ Kansas State University, Manhattan, Kansas 66506, USA

${ }^{60}$ Louisiana Tech University, Ruston, Louisiana 71272, USA

${ }^{61}$ University of Maryland, College Park, Maryland 20742, USA

${ }^{62}$ Boston University, Boston, Massachusetts 02215, USA

${ }^{63}$ Northeastern University, Boston, Massachusetts 02115, USA

${ }^{64}$ University of Michigan, Ann Arbor, Michigan 48109, USA

${ }^{65}$ Michigan State University, East Lansing, Michigan 48824, USA

${ }^{66}$ University of Mississippi, University, Mississippi 38677, USA

${ }^{67}$ University of Nebraska, Lincoln, Nebraska 68588, USA

${ }^{68}$ Princeton University, Princeton, New Jersey 08544, USA

${ }^{69}$ State University of New York, Buffalo, New York 14260, USA

${ }^{70}$ Columbia University, New York, New York 10027, USA

${ }^{71}$ University of Rochester, Rochester, New York 14627, USA

${ }^{72}$ State University of New York, Stony Brook, New York 11794, USA

${ }^{73}$ Brookhaven National Laboratory, Upton, New York 11973, USA

${ }^{74}$ Langston University, Langston, Oklahoma 73050, USA

${ }^{75}$ University of Oklahoma, Norman, Oklahoma 73019, USA

${ }^{76}$ Oklahoma State University, Stillwater, Oklahoma 74078, USA

${ }^{77}$ Brown University, Providence, Rhode Island 02912, USA

${ }^{78}$ University of Texas, Arlington, Texas 76019, USA

${ }^{79}$ Southern Methodist University, Dallas, Texas 75275, USA

${ }^{80}$ Rice University, Houston, Texas 77005, USA

${ }^{81}$ University of Virginia, Charlottesville, Virginia 22901, USA

${ }^{82}$ University of Washington, Seattle, Washington 98195, USA

(Received 16 June 2008; published 12 September 2008)

In this Letter we report on a search for long-lived particles that decay into final states with two electrons or photons. Such long-lived particles arise in a variety of theoretical models, such as hidden valleys and supersymmetry with gauge-mediated breaking. By precisely reconstructing the direction of the electromagnetic shower we are able to probe much longer lifetimes than previously explored. We see no evidence of the existence of such long-lived particles and interpret this search as a quasi model-independent limit on their production cross section, as well as a limit on a long-lived fourth generation quark. 
The standard model is surprisingly successful in describing phenomena observed at accelerators. One would expect, given its numerous theoretical shortcomings and the proliferation of searches for deviations from it, that a more general underlying theory would have been already revealed. It is therefore a possibility that the discovery of new physics eludes us because the new physics looks different from popular standard model extensions like minimal supersymmetry (SUSY).

In this Letter we search for pairs of electromagnetic (EM) showers from electrons or photons that originate from the same point in space, away from the $p \bar{p}$ interaction point. Such events can be a signature of a long-lived $b^{\prime}$ quark decaying into a $Z$ boson and a jet [1]. In models with gauge-mediated SUSY breaking [2] a long-lived neutralino with large higgsino component can decay into a $Z$ boson and a gravitino. In the hidden valley models [3], $v$ mesons can decay into electron pairs. In all of the above examples, a significant imbalance in transverse energy can be present due to $Z$ boson or $v$ hadron decays into neutrinos or lightest supersymmetric particles (LSP) that remain undetected.

A search for such long-lived particles at hadron colliders was performed by CDF [4] based on the reconstruction of lepton tracks from a secondary vertex. The sensitivity to large lifetimes in that search is limited by the difficulties in reconstructing tracks that originate far from the interaction point. In our analysis, we use the fine segmentation of the D0 detector to reconstruct the directions of the EM showers and use that to reconstruct the common vertex. This technique, used for the first time at a hadronic collider, allows us to probe dramatically longer decay lengths, albeit at the price of lower sensitivity to short lifetimes. Since we do not require the electron track to be reconstructed, our search results are also applicable for long-lived particles decaying into photons.

The data in this analysis were recorded with the D0 detector [5], which comprises an inner tracker, liquidargon or uranium calorimeters, and a muon spectrometer. The inner tracker is located in a $2 \mathrm{~T}$ superconducting solenoidal magnet and consists of silicon microstrip and scintillating-fiber trackers. It provides measurements of charged particle tracks up to pseudorapidity [6] of $|\eta| \approx$ 3.0. The calorimeter system consists of a central section (CC) covering $|\eta|<1.2$ and two end cap calorimeters extending the coverage to $|\eta| \approx 4$, all housed in separate cryostats [7]. The electromagnetic section of the calorimeter has four longitudinal layers and transverse segmentation of $0.1 \times 0.1$ in $\eta-\phi$ space (where $\phi$ is the azimuthal angle), except in the third layer, where it is $0.05 \times 0.05$. The central preshower (CPS) system is located between the solenoid and the CC calorimeter cryostat, covers $|\eta| \lesssim$ 1.2 , and provides measurement of EM shower position with a precision of about $1 \mathrm{~mm}$. The data for this study were collected between 2002 and summer 2006 using single EM triggers. The integrated luminosity [8] of the sample is $1100 \pm 70 \mathrm{pb}^{-1}$.
We select events with two EM clusters reconstructed in the central calorimeter with transverse momentum $p_{T}>$ $20 \mathrm{GeV}$ and $|\eta|<1.1$, with the shower shape consistent with that expected of a photon. EM clusters are required to be isolated in the calorimeter and tracker [9]. Each EM cluster is matched to the highest energy CPS cluster in an $\eta-\phi$ window centered on the line connecting the EM cluster and the primary vertex. The maximum possible distance of closest approach (DCA) to the beam line that can be reconstructed is determined by the size of the window and is approximately $16 \mathrm{~cm}$. Jets are reconstructed using the iterative midpoint cone algorithm [10] with a cone size of 0.5 . The missing transverse energy is determined from the energy deposited in the calorimeter for $|\eta|<4$ and is corrected for the EM and jet energy scales.

The D0 EM pointing algorithm [11] fits five shower position measurements (one in the CPS and four in the four EM layers of the central calorimeter) to a straight line which is assumed to be the EM object direction. The electron trajectory for energies above $20 \mathrm{GeV}$, which are of interest to this analysis, is very close to a straight line, which is defined by the energy-weighted EM cluster position $\left(x^{\mathrm{CAL}}, y^{\mathrm{CAL}}\right)$ and the DCA. The DCA reconstruction accuracy is about $2 \mathrm{~cm}$. The common vertex position in the $x y$ plane for two EM objects is the intersection of the two lines associated to them and is given by a solution of the system of two linear equations (see Fig. 1 for definitions of the trajectory and quantities below):

$$
\left(\begin{array}{ll}
-\Delta y_{1} & \Delta x_{1} \\
-\Delta y_{2} & \Delta x_{2}
\end{array}\right)\left(\begin{array}{l}
x \\
y
\end{array}\right)=\left(\begin{array}{l}
y_{1}^{\mathrm{CAL}} \Delta x_{1}-x_{1}^{\mathrm{CAL}} \Delta y_{1} \\
y_{2}^{\mathrm{CAL}} \Delta x_{2}-x_{2}^{\mathrm{CAL}} \Delta y_{2}
\end{array}\right)
$$

The determinant of this system, $D$, is proportional to the sine of the opening angle $\theta_{12}$ between the EM objects. The vertex transverse position resolution is inversely proportional to the determinant. Therefore, in the following we

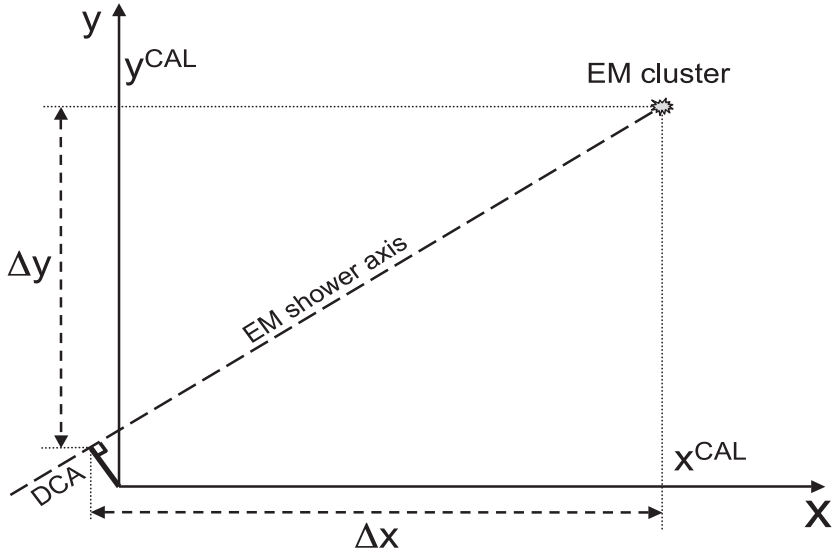

FIG. 1. Definition of the reconstructed EM particle trajectory. In the D0 coordinate system the equation of the trajectory is given by $\Delta x\left(y-y^{\mathrm{CAL}}\right)=\Delta y\left(x-x^{\mathrm{CAL}}\right)$. The distance from the beam line to the EM shower maximum $\sqrt{\left(x^{\mathrm{CAL}}\right)^{2}+\left(y^{\mathrm{CAL}}\right)^{2}}$ is typically around $90 \mathrm{~cm}$. 
consider events with $|D|>4000 \mathrm{~cm}^{2}$, which roughly corresponds to $\sin \theta_{12}>0.5$, and use the variable $R_{S}=$ $\pm \sqrt{x^{2}+y^{2}}\left(D / 1000 \mathrm{~cm}^{2}\right)$, which, while related to the reconstructed vertex radius, also takes into account its uncertainty. The sign of $R_{S}$ is given by the sign of the scalar product of the $\vec{p}_{T}$ of the pair of EM objects with the vector pointing from the origin to the vertex location of the two EM particles. To reduce the background we further require that at least one of the two EM objects has DCA $>2 \mathrm{~cm}$.

For vertices that originate from real particle decays, $R_{S}$ is positive, while its distribution for prompt electron or photon pairs is symmetrical around zero. The latter assumption was extensively checked with Monte Carlo (MC) simulation, a $Z \rightarrow e^{+} e^{-}$data sample (both electrons in the $Z \rightarrow e^{+} e^{-}$sample were required to have reconstructed tracks originating from the primary vertex), and a control sample of multi-jet events which have been selected exactly as the signal events except with an inverted tracker isolation requirement. Therefore, we estimate the background for positive values of $R_{S}$ by mirroring the negative part of the distribution.

The invariant mass $M$ of the two EM objects is corrected for the reconstructed vertex position, and the data are divided into three bins: $20<M<40,40<M<75$, and $M>75 \mathrm{GeV}$. The last bin is used for searches for the fourth generation $b^{\prime}$. The corresponding observed $R_{S}$ distribution is shown in Fig. 2. All mass bins are used for a quasi model-independent search for long-lived particles. We also examine events with $\not E_{T}>30 \mathrm{GeV}$ and $M>$ $20 \mathrm{GeV}$. No excess of events with positive $R_{S}$ values is present in the data (see Table I), so we proceed to set limits on new physics.

We use PYTHIA 6.319 [12] to generate events $p \bar{p} \rightarrow$ $b^{\prime} \bar{b}^{\prime} \rightarrow Z b Z b \rightarrow e^{+} e^{-}+X$. PYTHIA calculates production cross sections varying from 79.4 to $3.6 \mathrm{pb}$ as the $b^{\prime}$ mass

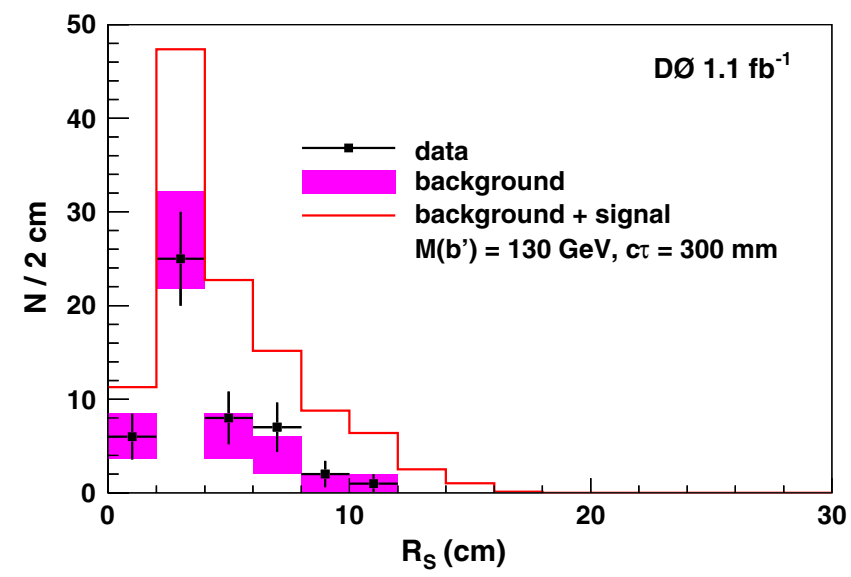

FIG. 2 (color online). Observed $R_{S}$ distribution for di-EM pairs with mass greater then $75 \mathrm{GeV}$ (black points), expected distribution from prompt sources with its uncertainty (shaded rectangles) and the expected distribution in presence of $b^{\prime}$ quark with mass of $130 \mathrm{GeV}$ and lifetime $c \tau=300 \mathrm{~mm}$ (solid line). changes from 100 to $190 \mathrm{GeV}$. The events are then processed through the GEANT-based [13] MC simulation, electronics and trigger simulation, and are reconstructed with the same reconstruction program as collision data. The expected $R_{S}$ distribution for a typical signal point is shown in Fig. 2. We use the efficiencies and acceptances obtained using the signal MC data for the quasi-model-independent search as well. The significant jet activity in these events gives a conservative estimate of the efficiency for SUSY scenarios and should be adequate for hidden valley models [14]. In order to study different masses of hypothetical resonances in addition to the samples above we also generated samples of $b^{\prime} \rightarrow v b$ for $v$ masses of 30 and $50 \mathrm{GeV}$. We find that the efficiency and acceptance for the MC events have no significant dependence on the masses of the $b^{\prime}$ and $v$. We set the $b^{\prime}$ mass to $150 \mathrm{GeV}$ and vary its lifetime $c \tau$ between 2 and $7000 \mathrm{~mm}$.

In Fig. 3 we display the limits on the production cross section of a long-lived particle times its branching fraction to decay into a pair of electrons. Limits were obtained from the $R_{S}$ distribution using the modified frequentist approach [15] as implemented in [16]. This method is based on a loglikelihood ratio (LLR) test statistic, and involves the calculation of confidence levels for the signal plus background and background-only (null) hypotheses (denoted by $C L_{s+b}$ and $C L_{b}$, respectively) by integrating the LLR distributions resulting from simulated pseudoexperiments. The upper limit on the cross section at the 95\% C.L. is defined as the cross section value for which the ratio $C L_{s}=C L_{s+b} / C L_{b}=0.05$. The systematic uncertainties were taken to be flat as a function of $R_{S}$. They include the uncertainty in electron or photon identification and triggering $(15 \%)$, uncertainty on MC simulation $(5 \%)$, and uncertainty on luminosity (6.1\%). At the $c \tau$ value of $100 \mathrm{~mm}$ we exclude at the $95 \%$ C.L. the production cross section times branching fraction of long-lived particles that decay into a pair of electrons or photons above 1.9, 10.2, 7.1, and $4.4 \mathrm{pb}$ for $\mathscr{E}_{T}>30 \mathrm{GeV}$ and $M>20 \mathrm{GeV}, 20<M<$ $40 \mathrm{GeV}, 40<M<75 \mathrm{GeV}$, and $M>75 \mathrm{GeV}$, respectively, (see Fig. 3).

Intersecting the cross section upper limits shown in Fig. 3(d) with the theoretical cross section of the production of the fourth generation $b^{\prime}$ quark [12] we compute limits on its lifetime as a function of its mass assuming it decays only into $Z b$. The limits are presented in Fig. 4, together with the exclusion region from the track-based

TABLE I. Observed number of events $\left(R_{S}>0 \mathrm{~cm}\right)$ and estimated background $\left(R_{S}<0 \mathrm{~cm}\right)$ for different selections.

\begin{tabular}{lcr}
\hline \hline \multicolumn{1}{c}{ Selection } & $R_{S}>0$ & $R_{S}<0$ \\
\hline $20<M<40 \mathrm{GeV}$ & 38 & 47 \\
$40<M<75 \mathrm{GeV}$ & 191 & 190 \\
$M>75 \mathrm{GeV}$ & 49 & 45 \\
$M>20 \mathrm{GeV}, \mathbb{E}_{T}>30 \mathrm{GeV}$ & 7 & 6 \\
\hline \hline
\end{tabular}




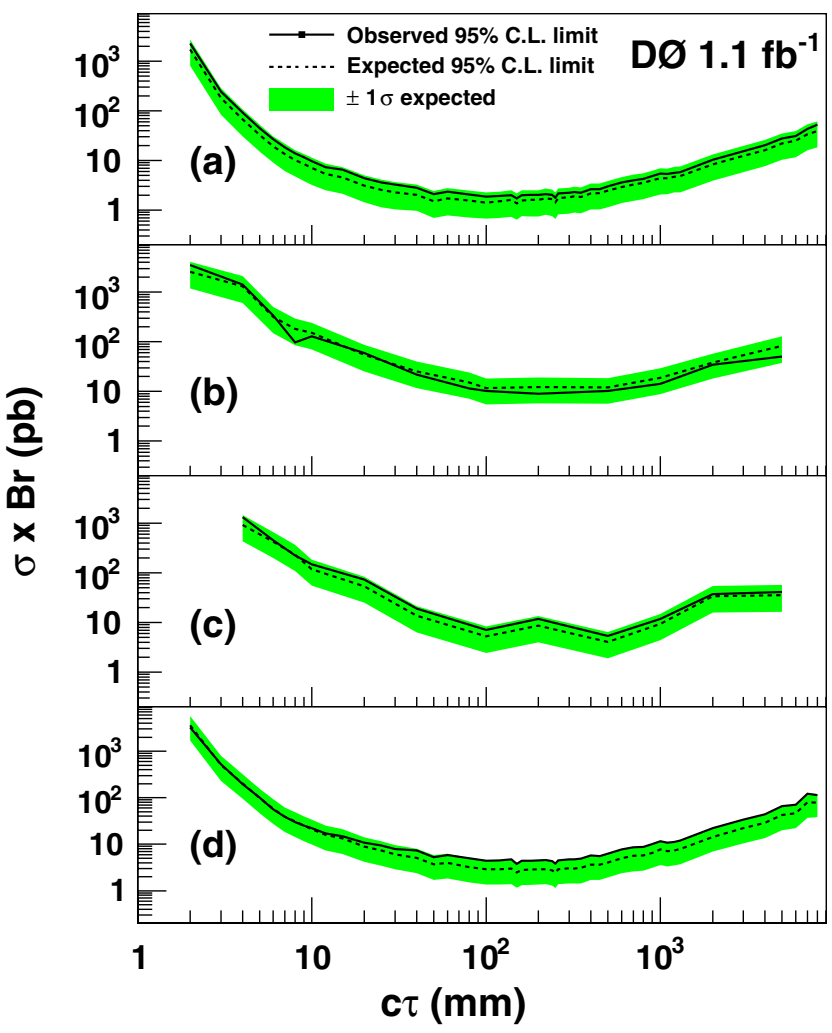

FIG. 3 (color online). Expected (dashed line) and observed (solid line) $95 \%$ C.L. upper limits on the cross section of a long-lived particle times the branching fraction of its decay to either a pair of electrons or photons for (a) $\mathbb{E}_{T}>30 \mathrm{GeV}$ and $M>20 \mathrm{GeV}$, (b) $20<M<40 \mathrm{GeV}$, (c) $40<M<75 \mathrm{GeV}$, and (d) $M>75 \mathrm{GeV}$. All observed upper limits are within 1 standard deviation (shaded band) from the expected limits.

CDF search [4]. The two search methods are complementary to each other.

To summarize, we have performed a search for longlived particles decaying into electron or photon pairs using

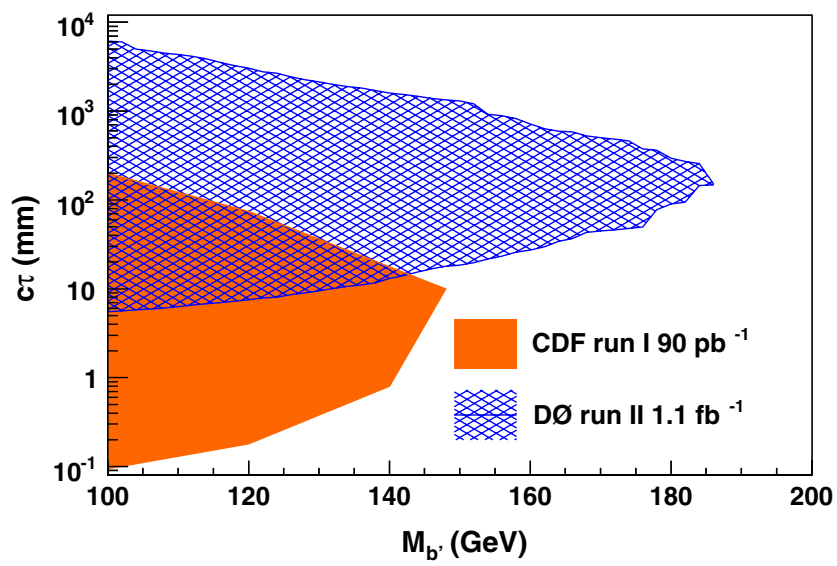

FIG. 4 (color online). $\quad 95 \%$ C.L. exclusion region of $b^{\prime}$ lifetime $(c \tau)$ vs mass for CDF run I [4] and current D0 result. a new method that allowed us to explore previously unreachable portions of the parameter space. We find no evidence for such particles and present the results as quasi model-independent limits on their production cross section and interpret them in the framework of a model with a long-lived $b^{\prime}$ quark [1].

We would like to thank Matt Strassler for many fruitful discussions.

We thank the staffs at Fermilab and collaborating institutions, and acknowledge support from the DOE and NSF (USA); CEA and CNRS/IN2P3 (France); FASI, Rosatom and RFBR (Russia); CNPq, FAPERJ, FAPESP and FUNDUNESP (Brazil); DAE and DST (India); Colciencias (Colombia); CONACyT (Mexico); KRF and KOSEF (Korea); CONICET and UBACyT (Argentina); FOM (The Netherlands); STFC (United Kingdom); MSMT and GACR (Czech Republic); CRC Program, CFI, NSERC and WestGrid Project (Canada); BMBF and DFG (Germany); SFI (Ireland); The Swedish Research Council (Sweden); CAS and CNSF (China); and the Alexander von Humboldt Foundation (Germany).

*Visitor from Augustana College, Sioux Falls, SD, USA.

${ }^{+}$Visitor from The University of Liverpool, Liverpool, United Kingdom.

${ }^{*}$ Visitor from ECFM, Universidad Autonoma de Sinaloa, Culiacàn, Mexico.

${ }^{\S}$ Visitor from II. Physikalisches Institut, Georg-AugustUniversity, Göttingen, Germany.

"Visitor from Helsinki Institute of Physics, Helsinki, Finland.

${ }^{\mathrm{T}}$ Visitor from Universität Bern, Bern, Switzerland.

**Deceased.

${ }^{++}$Visitor from Universität Zürich, Zürich, Switzerland.

[1] H. Frampton, P. Q. Hung, and M. Sher, Phys. Rep. 330, 263 (2000).

[2] S. Dimopoulos, S. Thomas, and J.D. Wells, Nucl. Phys. B488, 39 (1997); H. Baer, P. G. Mercadante, X. Tata, and Y.L. Wang, Phys. Rev. D 60, 055001 (1999); see also a review by G. F. Giudice and R. Rattazzi, in Perspectives on Supersymmetry, edited by G. L. Kane (World Scientific, Singapore, 1998), p. 355, and references therein.

[3] T. Han, Z. Si, K. Zurek, and M. Strassler, arXiv:0712.2041v1.M. Strassler and K. Zurek, Phys. Lett. B 651, 374 (2007).

[4] F. Abe et al. (CDF Collaboration), Phys. Rev. D 58, 051101 (1998).

[5] V. M. Abazov et al. (D0 Collaboration), Nucl. Instrum. Methods Phys. Res., Sect. A 565, 463 (2006).

[6] The D0 detector utilizes a right-handed coordinate system with the $z$ axis pointing in the direction of the proton beam and the $y$ axis pointing upwards. The azimuthal angle $\phi$ is defined in the $x y$ plane measured from the $x$ axis. The pseudorapidity is defined as $\eta=-\ln [\tan (\theta / 2)]$, where $\theta=\arctan \left(\sqrt{x^{2}+y^{2}} / z\right)$. 
[7] S. Abachi et al., Nucl. Instrum. Methods Phys. Res., Sect. A 338, 185 (1994).

[8] T. Andeen et al., Fermilab, Report No. FERMILAB-TM2365, 2007.

[9] For a description of the standard photon identification at D0 see, for example, V. M. Abazov et al. (D0 collaboration), Phys. Lett. B 659, 856 (2008).

[10] G. C. Blazey et al., Fermilab Report No. Pub-00/297 edited by U. Baur, R. K. Ellis, and D. Zeppenfeld (2000).

[11] D. Cutts and G. Landsberg, arXiv:hep-ph/0006162v2.
[12] T. Sjöstrand et al., Comput. Phys. Commun. 135, 238 (2001).

[13] R. Brun and F. Carminati, CERN Program Library Long Writeup W5013, 1993 (unpublished).

[14] M. Strassler (private communication).

[15] T. Junk, Nucl. Instrum. Methods Phys. Res., Sect. A 434, 435 (1999); A. Read, CERN, Report No. CERN 2000-005, 2000.

[16] W. Fisher, Fermilab, Report No. FERMILAB-TM-2386E, 2007. 